\title{
Clinical efficacy and safety of mecapegfilgrastim in small cell lung cancer as primary prophylaxis of neutropenia post chemotherapy: a retrospective analysis
}

\author{
Xiaodong Gu ${ }^{1,2,3} \wedge$, Yiping Zhang ${ }^{2,3}$ \\ ${ }^{1}$ The Second Clinical Medical College of Zhejiang Chinese Medical University, Hangzhou, China; ${ }^{2}$ Department of Thoracic Medical Oncology, \\ Cancer Hospital of the University of Chinese Academy of Sciences (Zhejiang Cancer Hospital), Hangzhou, China; ${ }^{3}$ Institute of Basic Medicine and \\ Cancer (IBMC), Chinese Academy of Sciences, Hangzhou, China \\ Contributions: (I) Conception and design: Y Zhang; (II) Administrative support: Y Zhang; (III) Provision of study materials or patients: Y Zhang; \\ (IV) Collection and assembly of data: X Gu; (V) Data analysis and interpretation: X Gu; (VI) Manuscript writing: Both authors; (VII) Final approval \\ of manuscript: Both authors. \\ Correspondence to: Yiping Zhang. Department of Thoracic Medical Oncology, Cancer Hospital of the University of Chinese Academy of Sciences \\ (Zhejiang Cancer Hospital), No.1 Banshan East Street, Gongshu District, Hangzhou 330022, China. Email: zyp@medmail.com.cn.
}

Background: Neutropenia is the most common adverse reaction seen in small cell lung cancer after
chemotherapy. Febrile neutropenia (FN) leads to an increase in hospitalizations and may even be life-
threatening. This paper aims to investigate the efficacy and adverse reactions of mecapegfilgrastim in the
primary prophylaxis of neutropenia in patients with small cell lung cancer after receiving intermediate risk
chemotherapy with at least one patient risk factor. Methods: The clinical records of 106 patients with small cell lung cancer admitted to Zhejiang Cancer Hospital from June 2019 to January 2021 were retrospectively analyzed. Patients were divided into a mecapegfilgrastim [pegylated recombinant human granulocyte colony stimulating factor (PEG-rhG-CSF)] group and control group, each with 53 patients. The mecapegfilgrastim group received subcutaneous injection of mecapegfilgrastim 24 hours after the first cycle of chemotherapy, while the control group did not receive this. The Chi-square $\left(\chi^{2}\right)$ test or Fisher exact test were used to compare the incidence of neutropenia, $\mathrm{FN}$, and the proportion of patients administrated with full dose chemotherapy in the two groups after the first cycle of chemotherapy. Data on adverse events after mecapegfilgrastim were also collected.

Results: After the first cycle of chemotherapy, the incidence of neutropenia in the mecapegfilgrastim group was significantly lower than that in the control group $(\mathrm{P}=0.001)$ and the incidence of $\mathrm{FN}$ in the mecapegfilgrastim group was lower than that in the control group $(\mathrm{P}=0.118)$. The proportion of patients administrated with full-dose chemotherapy in the mecapegfilgrastim group was significantly higher than that in the control group $(\mathrm{P}=0.001)$. The main adverse reactions to mecapegfilgrastim were muscle pain, fever, and fatigue.

Conclusions: After receiving intermediate risk chemotherapy, the incidence of neutropenia was significantly reduced by the primary prophylaxis of mecapegfilgrastim in patients with small cell lung cancer. The adverse events of mecapegfilgrastim were mild and tolerable, and included muscle pain, fever, and fatigue.

Keywords: Pegylated recombinant human granulocyte colony stimulating factor (PEG-rhG-CSF); mecapegfilgrastim; primary prophylaxis; chemotherapy; neutropenia

Submitted May 10, 2021. Accepted for publication Jun 24, 2021.

doi: 10.21037/apm-21-1400

View this article at: https://dx.doi.org/10.21037/apm-21-1400

\footnotetext{
$\wedge$ ORCID: 0000-0001-5776-6267.
} 


\section{Introduction}

The incidence and mortality of lung cancer, including non-small cell lung cancer (NSCLC) and small cell lung cancer (SCLC), rank at the forefront of malignant tumors in China (1). SCLC accounts for about $13-15 \%$ of lung cancers, and most patients are in the extensive stage at initial diagnosis, with only one third in the limited stage (2). Platinum-based chemotherapy is still the main treatment for SCLC patients in the extensive stage because of its high sensitivity, while concurrent radiotherapy and chemotherapy are the main treatment for patients in the limited stage. Myelosuppression is a common complication of chemotherapy, especially when a decrease in the absolute neutrophil count (ANC) in peripheral blood occurs. This not only increases the risk of infection and death, but also reduces the dose of chemotherapy drugs, prolongs the intermittent period of chemotherapy, and may even cause it to be terminated. At present, recombinant human granulocyte colony stimulating factor (rhG-CSF) is the most used myeloid growth factor (MGF) in clinical practice, but continuous daily administration is required for its short half-life, which may be inconvenient, and the compliance of patients is poor (3). Pegylated recombinant human granulocyte colony stimulating factor (PEG-rhG-CSF) is a long-acting G-CSF and has a longer half-life and a more stable plasma concentration than rhG-CSF. Moreover, PEG-rhG-CSF is unlikely to be catalyzed by enzymes due to its high molecular weight and strong biological stability and is unlikely to produce neutralizing antibodies with reduced immunogenicity and antigenicity (4). Due to its long half-life, subcutaneously administration of PEG-rhG-CSF is required only once per 24-h after each chemotherapy cycle to prevent neutropenia (5), which can increase compliance, reduce the length of hospital stay, and improve quality of life during the entire chemotherapy process. According to the NCCN Guidelines for Hematopoietic Growth Factors, G-CSF should be administered prophylactically if the risk of febrile neutropenia $(\mathrm{FN})$ is $>20 \%$, and if the risk is intermediate (10-20\%), the risk factors of individual patients are required to be considered. This study explored the efficacy and adverse reactions of PEG-rhG-CSF primarily administered after chemotherapy in patients with SCLC who had at least one patient risk factor for $\mathrm{FN}$.

We present the following article in accordance with the STROBE reporting checklist (available at https://dx.doi. org/10.21037/apm-21-1400).

\section{Methods}

\section{Patients}

The clinical records of 106 patients with SCLC who attended the Thoracic Medical Oncology of Zhejiang Cancer Hospital between June 2019 and January 2021 were retrospectively selected.

The inclusion criteria were all of the following: Patients with pathologically confirmed SCLC; elderly patients in the limited stage with indications for chemotherapy (but intolerable to cisplatin) or patients in the extensive stage with indications for first-line chemotherapy, with all chemotherapy regimens being etoposide combined with carboplatin; the presence of at least one risk factor; and a performance status (PS) score of $0-2$; leukocytes $\geq 3.5 \times 10^{9} / \mathrm{L}$, neutrophils $\geq 1.8 \times 10^{9} / \mathrm{L}$, platelets $\geq 120 \times 10^{9} / \mathrm{L}$, and hemoglobin $\geq 100 \mathrm{~g} / \mathrm{L}$ before chemotherapy.

Following the Chinese Society of Clinical Oncology Guidance Working Committee (6), risk factors were considered as any of the following: patients age $>65$ years receiving full-dose chemotherapy; previously received radiotherapy or chemotherapy; persistent neutropenia; bone marrow infiltration; recent surgery and/or open wound; abnormal liver function (bilirubin $>34.2 \mu \mathrm{mol} / \mathrm{L}$ ); abnormal renal function (creatinine clearance rate $<50 \mathrm{~mL} / \mathrm{min}$ ); previous occurrence of $\mathrm{FN}$; or malignant hemolymphatic system disease.

Ethical approval and patient consent were waived by the Ethics Committee at Zhejiang Cancer Hospital in view of the retrospective nature of the study. All procedures performed in this study involving human participants were in accordance with the Declaration of Helsinki (as revised in 2013).

\section{Treatment}

A chemotherapy regimen using etoposide $\left(100 \mathrm{mg} / \mathrm{m}^{2}\right.$, given for three consecutive days) combined with carboplatin (AUC =5, day 1), was administered once every three weeks. Patients were equally divided into a mecapegfilgrastim group containing 53 patients who were injected subcutaneously with mecapegfilgrastim $6 \mathrm{mg}$ once 24 hours after the first cycle of chemotherapy, and a control group containing 53 patients not receiving it. Mecapegfilgrastim was provided by the Jiangsu Hengrui Medicine Co., Ltd. (Lianyungang, China). 


\section{Observation indicators}

Blood samples were collected for all patients on the seventh day after the first cycle of chemotherapy, and the neutrophil count and levels of neutropenia were calculated. The grade of neutropenia was evaluated according to the WHO Acute and Subacute Toxicity Criteria of Anti-cancer Drugs into grade $0-4$ (grade $0: \geq 2.0 \times 10^{9} / \mathrm{L}$, grade $1:(1.5-1.9) \times 10^{9} / \mathrm{L}$, grade $2:(1.0-1.4) \times 10^{9} / \mathrm{L}$, grade $3:(0.5-0.9) \times 10^{9} / \mathrm{L}$, and grade $\left.4:<0.5 \times 10^{9} / \mathrm{L}\right)$. Between four and six chemotherapy cycles were monitored and whether the chemotherapy was completed in full dose was recorded. The proportion of patients with full dose chemotherapy administration was calculated as were adverse events.

\section{Statistical analysis}

SPSS 26.0 was used to perform statistical analysis. Categorical variables were reported as numbers (percentages) and the difference between groups was tested with the $\chi^{2}$ test or Fisher exact test. $\mathrm{P}<0.05$ was considered statistically significant.

\section{Results}

\section{Patient characteristics}

The mecapegfilgrastim group comprised 38 patients younger than 70 years; 43 patients were male and 10 were female; 33 patients had an Eastern Cooperative Oncology Group (ECOG) performance status score (PS) of 0, 19 had an ECOG PS of 1, and one patient had an ECOG PS of 2; 10 patients were in limited stage and 43 patients were in extensive stage; 13 patients received radiotherapy previously; and 15 patients were diagnosed with bone metastasis upon treatment. In the control group, there were 29 patients younger than 70 years; 45 patients were male and 8 were female; 35 patients had an EOCG PS of 0 and 18 had an EOCG PS of 1; 13 patients were in limited stage and 40 were in extensive stage; 12 patients had received previous radiotherapy; and 18 patients were diagnosed with bone metastasis upon treatment. As there was no significant difference between the two groups by $\chi^{2}$ test or Fisher exact test $(\mathrm{P}>0.05)$, the baseline characteristics between two groups were comparable (Table 1).

\section{Bone marrow suppression after the first cycle of chemotherapy}

In the mecapegfilgrastim group, 10 patients (19\%) experienced grade I/II neutropenia and 5 (9\%) experienced grade III/IV neutropenia, while in the control group, 20 patients (38\%) experienced grade I/II neutropenia and 21 (40\%) experienced grade III/IV neutropenia. After the first cycle of chemotherapy, there was a statistically significant difference in the incidence of neutropenia between the two groups $(\mathrm{P}=0.001)$ (Table 2).

\section{Incidence of $F N$}

After the first round of chemotherapy $\mathrm{FN}$ was seen in four patients $(8 \%)$ in the control group and none in the mecapegfilgrastim group. There was no significant difference in the incidence of FN between the two groups ( $0 \%$ vs. $8 \%, \mathrm{P}=0.118)$.

\section{Chemotherapy dosage}

The dosage of chemotherapy drugs was reduced in three of five patients with grade III/IV neutropenia in the mecapegfilgrastim group from the second cycle, and the proportion of patients treated with full-dose chemotherapy was $94 \%$. In the control group, 18 of 21 patients with grade III/IV neutropenia started to reduce dosage from the second cycle, and the proportion of patients treated with full-dose chemotherapy was $66 \%$. There was a statistically significant difference in the proportion of patients treated with full dose chemotherapy between the two groups ( $94 \%$ vs. $66 \%, \mathrm{P}<0.001)$.

\section{Adverse reactions to mecapegfilgrastim}

In the mecapegfilgrastim group, nine patients $(17 \%, 9 / 53)$ developed muscle soreness after using mecapegfilgrastim, of which eight patients had mild soreness and one had severe muscle pain, which was relieved by using non-steroidal anti-inflammatory drugs. Three cases $(6 \%)$ in this group developed fever below $38.3{ }^{\circ} \mathrm{C}$ which was reduced with non-steroidal anti-inflammatory drugs or physical cooling. Five patients $(9 \%)$ complained of fatigue, and one (2\%) presented with hoarseness secondary to vocal cord edema caused by mecapegfilgrastim, and the symptoms disappeared after the regimen was changed to short-acting rhG-CSF. Finally, three patients $(6 \%)$ reported mild nausea, which was relieved spontaneously without intervention (Table 3).

\section{Discussion}

Chemotherapy is an important treatment for SCLC, 
Table 1 Baseline characteristics of patients

\begin{tabular}{|c|c|c|c|c|}
\hline Characteristics & Total $(n=106)$ & Mecapegfilgrastim group $(n=53)$ & Control group $(n=53)$ & $P$ value \\
\hline$<70$ & $67(63.2)$ & $38(71.7)$ & $29(54.7)$ & 0.107 \\
\hline$\geq 70$ & $39(36.8)$ & $15(28.3)$ & $24(45.3)$ & \\
\hline \multicolumn{5}{|l|}{ Sex, n (\%) } \\
\hline Female & $18(17.0)$ & $10(18.9)$ & $8(15.1)$ & \\
\hline \multicolumn{5}{|l|}{ EOCG PS, n (\%) } \\
\hline 0 & $68(64.2)$ & $33(62.3)$ & $35(66.0)$ & 0.804 \\
\hline 1 & $37(34.9)$ & $19(35.8)$ & $18(34.0)$ & \\
\hline Limited & $23(21.7)$ & $10(18.9)$ & $13(24.5)$ & 0.480 \\
\hline Extensive & $83(78.3)$ & $43(81.1)$ & $40(75.5)$ & \\
\hline \multicolumn{5}{|c|}{ Prior radiotherapy, n (\%) } \\
\hline Yes & $25(23.6)$ & $13(24.5)$ & $12(22.6)$ & 0.819 \\
\hline No & $81(76.4)$ & $40(75.5)$ & $41(77.4)$ & \\
\hline \multicolumn{5}{|c|}{ Bone metastases, n (\%) } \\
\hline Yes & $33(31.1)$ & $15(28.3)$ & $18(34.0)$ & 0.529 \\
\hline No & $73(68.9)$ & $38(71.7)$ & $35(66.0)$ & \\
\hline
\end{tabular}

Table 2 Bone marrow suppression after the first cycle of chemotherapy

\begin{tabular}{lccl}
\hline Neutropenia classification & Mecapegfilgrastim group & Control group & $P$ \\
\hline Grade I/II neutropenia & $10(19 \%)$ & $20(37.7 \%)$ & $<0.001$ \\
Grade III/IV neutropenia & $5(9 \%)$ & $21(39.6 \%)$ & $12(22.6 \%)$ \\
Grade 0 or normal & $38(72 \%)$ & & \\
\hline
\end{tabular}

Table 3 Adverse events after using mecapegfilgrastim

\begin{tabular}{lcc}
\hline Adverse events $(\mathrm{n}=21)$ & Grade & Number (percent) \\
\hline Musculoskeletal pain & Mild & $8(15 \%)$ \\
& Moderate & $1(2 \%)$ \\
Fever & Mild & $3(6 \%)$ \\
Fatigue & Mild & $5(9 \%)$ \\
Hoarseness & mild & $1(2 \%)$ \\
Nausea & mild & $3(6 \%)$ \\
\hline
\end{tabular}

and myelosuppression is its most common adverse reaction. Some patients also suffer from $\mathrm{FN}$, which as a severe hematological toxicity may lead to an increase in hospitalizations and even be life-threatening (7). While PEG-rhG-CSF can be used for the primary prophylaxis of neutropenia after the first cycle of chemotherapy (5), it can also be used as a means of secondary prophylaxis in patients who present with $\mathrm{FN}$ or dose-limiting neutropenia during previous cycles of chemotherapy (8).

According to the NCCN Guidelines for Hematopoietic 
Growth Factors, the combination of etoposide and carboplatin is a regimen posed intermediate risk (10-20\%) for FN in patients with SCLC (9). The prophylactical administration of G-CSF is recommended in high-risk (>20\%) regimens regardless of the treatment purpose, while this can be considered in $\mathrm{FN}$ intermediate risk (10-20\%) regimens depending on the risk factors of the patient (10). This study explored the efficacy and safety of mecapegfilgrastim as primary prophylaxis in SCLC patients treated with intermediate risk chemotherapy who had at least one risk factor.

The results showed that after the first cycle of chemotherapy, the incidence of grade I/II and grade III/ IV neutropenia in the mecapegfilgrastim group was significantly lower than that of the control group and the proportion of patients treated with full dose chemotherapy in the mecapegfilgrastim group was significantly higher than in the control group. These results are similar to those of Zhou, who found that the incidence of grade III/ IV neutropenia and FN in NSCLC patients was $14.6 \%$ and $8 \%$, respectively (5). The incidence of FN between two groups were not significantly different. The adverse events of mecapegfilgrastim are mainly muscle pain, fever, and fatigue, which are similar to those seen recorded in multiple clinical studies carried out on breast cancer and $\operatorname{NSCLC~}(5,8,11)$.

Since this is a retrospective study, there are limitations in sample size, statistical biases, and differences in patient selection and further clinical studies or largescale prospective randomized controlled clinical trials are required to verify the results.

In conclusion, primary prophylaxis using mecapegfilgrastim in SCLC in a real-world setting can significantly reduce the incidence of neutropenia after intermediate risk chemotherapy. The adverse events of mecapegfilgrastim are mild and tolerable.

\section{Acknowledgments}

Funding: None.

\section{Footnote}

Reporting Checklist: The authors have completed the STROBE reporting checklist. Available at https://dx.doi. org/10.21037/apm-21-1400

Data Sharing Statement: Available at https://dx.doi. org/10.21037/apm-21-1400
Conflicts of Interest: Both authors have completed the ICMJE uniform disclosure form (available at https://dx.doi. org/10.21037/apm-21-1400). The authors have no conflicts of interest to declare.

Ethical Statement: The authors are accountable for all aspects of the work in ensuring that questions related to the accuracy or integrity of any part of the work are appropriately investigated and resolved. Ethical approval and patient consent were waived by the Ethics Committee at Zhejiang Cancer Hospital in view of the retrospective nature of the study. The study was conducted in accordance with the Declaration of Helsinki (as revised in 2013).

Open Access Statement: This is an Open Access article distributed in accordance with the Creative Commons Attribution-NonCommercial-NoDerivs 4.0 International License (CC BY-NC-ND 4.0), which permits the noncommercial replication and distribution of the article with the strict proviso that no changes or edits are made and the original work is properly cited (including links to both the formal publication through the relevant DOI and the license). See: https://creativecommons.org/licenses/by-nc-nd/4.0/.

\section{References}

1. Sung H, Ferlay J, Siegel RL, et al. Global Cancer Statistics 2020: GLOBOCAN Estimates of Incidence and Mortality Worldwide for 36 Cancers in 185 Countries. CA Cancer J Clin 2021;71:209-49.

2. Ganti AK, Zhen W, Kessinger A. Limited-stage smallcell lung cancer: therapeutic options. Oncology (Williston Park) 2007;21:303-12; discussion 312, 315-8, 323.

3. Chen J, Pan Y. The safety and clinical efficacy of recombinant human granulocyte colony stimulating factor injection for colon cancer patients undergoing chemotherapy. Rev Assoc Med Bras (1992) 2017;63:1061-4.

4. Yang BB, Kido A. Pharmacokinetics and pharmacodynamics of pegfilgrastim. Clin Pharmacokinet 2011;50:295-306.

5. Zhou C, Huang Y, Wang D, et al. A Randomized Multicenter Phase III Study of Single Administration of Mecapegfilgrastim (HHPG-19K), a Pegfilgrastim Biosimilar, for Prophylaxis of Chemotherapy-Induced Neutropenia in Patients With Advanced Non-SmallCell Lung Cancer (NSCLC). Clin Lung Cancer 2016;17:119-27. 
6. Chinese Society of Clinical Oncology Guidance Working Committee. Guidelines for standardizedmanagement of neutropenia induced by chemotherapy and radiotherapy. Zhonghua Zhong Liu Za Zhi 2017;39:868-78.

7. Zuo JM, Xia RX. The efficacy of combining rh-GCSF and antibiotics in patients with acute lymphoblastic leukemia. Chinese Journal of Gerontology 2016;36:5064-6.

8. Wang T, Wu B, Hu X, et al. A randomized multicenter phase II trial of mecapegfilgrastim single administration versus granulocyte colony-stimulating growth factor on treating chemotherapy-induced neutropenia in breast cancer patients. Ann Transl Med 2019;7:196.

9. Kosmidis PA, Samantas E, Fountzilas G, et al. Cisplatin/ etoposide versus carboplatin/etoposide chemotherapy and

Cite this article as: Gu X, Zhang Y. Clinical efficacy and safety of mecapegfilgrastim in small cell lung cancer as primary prophylaxis of neutropenia post chemotherapy: a retrospective analysis. Ann Palliat Med 2021;10(7):7841-7846. doi: 10.21037/ apm-21-1400 irradiation in small cell lung cancer: a randomized phase III study. Hellenic Cooperative Oncology Group for Lung Cancer Trials. Semin Oncol 1994;21:23-30.

10. Becker PS, Griffiths EA, Alwan LM, et al. NCCN Guidelines Insights: Hematopoietic Growth Factors, Version 1.2020. J Natl Compr Canc Netw 2020;18:12-22.

11. Xu F, Zhang Y, Miao Z, et al. Efficacy and safety of mecapegfilgrastim for prophylaxis of chemotherapyinduced neutropenia in patients with breast cancer: a randomized, multicenter, active-controlled phase III trial. Ann Transl Med 2019;7:482. Erratum in: Ann Transl Med 2020;8:660.

(English Language Editor: B. Draper) 